\title{
A Novel Linear PID Controller for an Upper Limb Exoskeleton
}

\author{
Wen Yu and Jacob Rosen
}

\begin{abstract}
An upper limb exoskeleton is a wearable robotic system that is physically linked to the arm of the human operators and its seven actuated degrees of freedom (DOF) match the seven DOF of the human arm. The stability of such a system is critical given the proximity of its human operator. A new PID controller is developed which guarantee asymptotic stability for this class of robotic manipulators. A simulation was used to assess the system performance given the theoretical results of the controller's parameters with a unique exoskeleton system (EXO-UL7). The simulation also verify the semi-global asymptotic stability of the system. The proposed methodology eliminates the need of the system's dynamics model for the purpose of designing the controller. It provides an analytical tool for the controller design that is traditionally preformed experimentally (parameter tuning).
\end{abstract}

\section{INTRODUCTION}

Exoskeletons are wearable robots, which are worn by the human operators as orthotic devices. The exoskeleton links, joints and workspace correspond to those of the human body. The system may be used as a human input device for teleoperation, human-amplifier, and physical therapy modality as part of the rehabilitation process [12]. A wide variety of exoskeleton systems both for upper limbs [5][6][17][22][23][35][38][33] and lower limbs $[11][10][8][15][16][3][4][9][14][42]$ with various humanmachine interfaces have been developed (for review see [7][11][29][41]).

Although great progress has been made in a centurylong effort to design and implement robotic exoskeletons, many design challenges continue to limit the performance of the system. One of the limiting factors is the lack of simple and effective control systems for the exoskeleton [13][39]. Proportional derivative (PD) control is the simplest scheme that may be used to control robot manipulators. It is known that bounded stability can be guaranteed with a positive PD gains controller for system regulation [37]. The robotic system performance which utilizes a PD controller is limited unless gravity compensation is applied, which requires a model of the system's dynamic [40][36] [18], [26]. Nonlinear PD controllers can also achieve asymptotic stability, such as PD control with time-varying gains [34], nonlinear modification [24], and sliding mode compensation [25].

Given the complexity of the of the exoskeleton as 7 DOF system a PID controller may be an alternative to PD

Wen Yu is with the Departamento de Control Automatico, CINVESTAVIPN, Av.IPN 2508, México D.F., 07360, México. Jacob Rosen is with the Department of Computer Engineering, University of California - Santa Cruz, 1156 High Street, Santa Cruz, CA 95064, USA. e-mail: rosen@ucsc.edu. URL: http://bionics.soe.ucsc.edu/

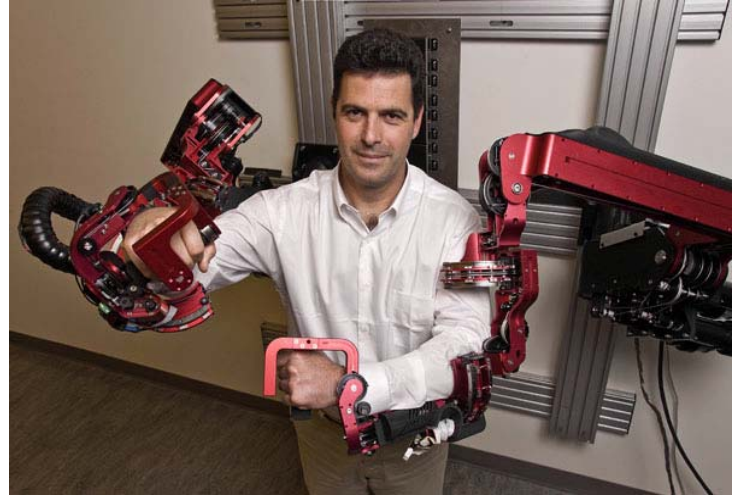

Fig. 1. The 7 DOF upper limb exoskeleton (EXO-UL7)

controller along with gravity compensation. The position error caused by gravitational torques can be reduced by introducing an integral component to the PD control. In order to assure asymptotic stability, several components were previously added to the classical linear PID controllers for example: forth order filter [24], nonlinear derivative term [2], nonlinear integral term (saturated function) [19], input saturation, and nonlinear observer [1]. Linear PID is the simplest and the most popular industrial controller, since tuning its internal parameters does not require a model of the plant and can be performed experimentally. Lyapunov function was previously used for the tuning procedure of a linear PID, however a the inertia matrix and the gravitational torque vector of the system have to be clearly defined [21], [20]. The stability of linear PID control was studied, where the robot dynamic is rewritten in decoupled linear system and bounded nonlinear system [30], however asymptotic stability was not achieved.

The aim of this research effort is to design PID controller for a 7 DOF upper limb exoskeleton [27][28][31][32], see Figure 1. The semiglobal asymptotic stability is proven along with a new approach for tuning the parameters of PID controller.

\section{Dynamic Model of the EXO-Ul7 Upper Limb EXOSKELETON}

The direct kinematics of the 7-DOF exoskeleton robot is derived based on the modified Denavit-Hartenberg (DH) convention. The frame assignments are depicted in Figure 2. The DH parameters of system are listed [27]. The homogeneous transformation matrix $T_{0}^{i}$ as a function of the four DH parameters per link $\left(a_{i}, d_{i}, \alpha_{i}\right.$, and $\left.\theta_{i}\right)$ defines the 


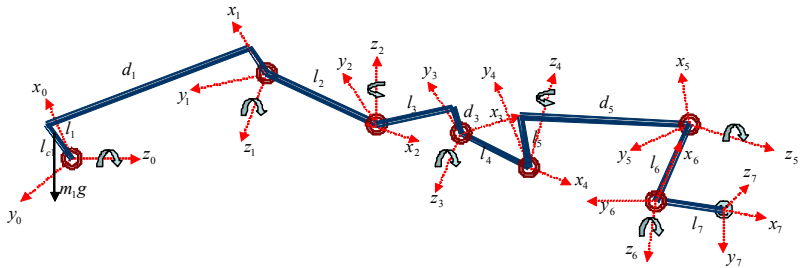

Fig. 2. Frames of the 7 DOF exoskeleton

transformation between two consecutive frames

$$
T_{0}^{i}=\left[\begin{array}{cccc}
c_{\theta_{i}} & -s_{\theta_{i}} c_{\alpha_{i}} & s_{\theta_{i}} s_{\alpha_{i}} & a_{i} c_{\theta_{i}} \\
s_{\theta_{i}} & c_{\theta_{i}} c_{\alpha_{i}} & -c_{\theta_{i}} s_{\alpha_{i}} & a_{i} s_{\theta_{i}} \\
0 & s_{\alpha_{i}} & c_{\alpha_{i}} & d_{i} \\
0 & 0 & 0 & 1
\end{array}\right]
$$

where $c_{\theta_{i}}=\cos \theta_{i}, s_{\theta_{i}}=\sin \theta_{i}, \theta_{i}$ is the rotation angle of the $\mathrm{i}$-th frame, $a_{i}$ is the distance between the axes $z_{i-1}$ and $z_{i}$, and is measured along the axis $x_{i}, d_{i}$ is the distance between the origin $o_{i-1}$ and the intersection of the $x_{i}$ axis with $z_{i-1}$, measured along the $z_{i-1}$ axis, $\alpha_{i}$ is the angle between the axes axes $z_{i-1}$ and $z_{i}$, measured in a plane normal to $x_{i}, \theta_{i}$ is the angle between the axes axes $x_{i-1}$ and $x_{i}$, measured in a plane normal to $z_{i-1}$.

Homogeneous transformation matrix describing the position and orientation of frame $i$ with respect to frame 0 is defined by $T_{0}^{i}$ as

$$
T_{0}^{i}=T_{0}^{1} T_{1}^{2} \cdots T_{i-1}^{i}, \quad i=1,2, \cdots 7
$$

The Jacobian of joint $o_{i}$ in the base frame is

$$
J_{\omega}=\left[z_{0}, z_{1}, \cdots z_{i-1}\right]
$$

where $z_{i-1}$ w.r.t. the base frame are given by the first three elements in the third column of $T_{0}^{i}$. From D-H convention

$$
T_{0}^{n}=\left[\begin{array}{cc}
R_{0}^{n} & R_{0}^{i} o_{i}^{n}+R_{0}^{i-1} o_{i-1}^{i}+o_{0}^{i-1} \\
0 & 1
\end{array}\right]
$$

where $o_{i}$ is given by the first three elements of the fourth column of $T_{0}^{i}$. If joint $j$ is revolute, $i$-th column of $J_{v}$ is

$$
\begin{aligned}
& J_{v}=\left[J_{v 1} \cdots J_{v n}\right] \\
& J_{v_{i}}=z_{i-1} \times\left(o_{j}-o_{i-1}\right)
\end{aligned}
$$

The dynamics of exoskeleton robot can be defined based on translational kinetic energy, rotational kinetic energy, potential energy and friction. Translational kinetic energy is defined as

$$
K_{T}=\frac{1}{2} \dot{q}^{T}\left[\sum_{i=1}^{n} m_{i} J_{v_{i}}^{T}(q) J_{v_{i}}(q)\right] \dot{q}
$$

Rotational kinetic energy is defined as

$$
K_{R}=\frac{1}{2} \sum_{i=1}^{n}\left(\omega_{0}^{i}\right)^{T} I_{i} \omega_{0}^{i}=\frac{1}{2} \dot{q}^{T}\left[\sum_{i=1}^{n} J_{\omega_{i}}^{T} I_{i} J_{\omega i}\right] \dot{q}
$$

where $I_{i}$ is the inertia tensor. The potential energy $U$ is defined as

$$
U=\sum_{i=1}^{n} m_{i} g h_{0}^{i}
$$

The equation of motion defining the dynamics of exoskeleton robots are derived based on the Euler-Lagrange formulation as

$$
M(q) \ddot{q}+C(q, \dot{q}) \dot{q}+g(q)=u
$$

where $q \in R^{n}$ represents the link positions. $n$ is joint number, for our 7-DOF exoskeleton robot $n=7 . M(q)$ is the inertia matrix, $C(q, \dot{q})=\left\{c_{k j}\right\}$ represents centrifugal force, $c_{k j}=\sum_{i=1}^{n} c_{i j k} \dot{q}_{i}, k, j=1 \cdots n, c_{i j k}$ is Christoffel symbols [37]

$$
c_{i j k}=\frac{1}{2}\left(\frac{\partial d_{k j}}{\partial q_{i}}+\frac{\partial d_{k i}}{\partial q_{j}}-\frac{\partial d_{i j}}{\partial q_{k}}\right)
$$

$g(q)$ is the joint torques vector due to the gravitational loads, $g(q)=\frac{\partial}{\partial q} U(q)$.

The 7-DOF exoskeleton satisfies the following well known properties .

P1. The inertia matrix $M(q)$ is symmetric positive definite, and

$$
0<\lambda_{m}\{M(q)\} \leq\|M\| \leq \lambda_{M}\{M(q)\} \leq \beta, \quad \beta>0
$$

where $\lambda_{M}\{A\}$ and $\lambda_{m}\{A\}$ are the maximum and minimum eigenvalues of the matrix $A$.

P2. For the Centrifugal and Coriolis matrix $C(q, \dot{q})$, there exists a number $k_{c}>0$ such that

$$
\|C(q, \dot{q}) \dot{q}\| \leq k_{c}\|\dot{q}\|^{2}, \quad \beta>0
$$

and $\dot{M}(q)-2 C(q, \dot{q})$ is skew symmetric, i.e.

$$
x^{T}[\dot{M}(q)-2 C(q, \dot{q})] x=0
$$

also

$$
\dot{M}(q)=C(q, \dot{q})+C(q, \dot{q})^{T}
$$

P3. The gravitational torques vector $g(q)$ is Lipschitz

$$
\|g(x)-g(y)\| \leq k_{g}\|x-y\|
$$

\section{Semi-Global Asymptotic Stability of A LINEAR PID CONTROL}

The position control objective is to evaluate the torques that have to be applied to the applied to the joints of the robot such that the difference between the actual joint angles and the desired joint angles (joint angles error) approach asymptotically to a constant. Given a desired joint angle vector $q^{d} \in R^{n}$, semi-global asymptotic stability of robot control is to design for an input torque vector $u$ in (9) which generates regulation error

$$
\tilde{q}=q^{d}-q
$$

$\tilde{q} \rightarrow 0$ and $\tilde{q} \rightarrow 0$ when the initial conditions are in arbitrary large domain of attraction.

The classical linear PID control law is defined as

$$
u=K_{p} \tilde{q}+K_{i} \int_{0}^{t} \tilde{q}(\tau) d \tau+K_{d} \tilde{q}
$$


where $K_{p}, K_{i}$ and $K_{d}$ are proportional, integral and derivative gains of the PID controller, respectively. Because $\dot{q}^{d}=0$, and $\dot{\tilde{q}}=-\dot{q}$, the PID control law can be expressed as

$$
\begin{aligned}
& u=K_{p} \tilde{q}-K_{d} \dot{q}+\xi \\
& \dot{\xi}=K_{i} \tilde{q}, \quad \xi(0)=\xi_{0}
\end{aligned}
$$

Using decoupled linear control (18) approach for which $K_{p}, K_{i}$ and $K_{d}$ are positive definite diagonal matrices, the closed-loop system of the robot (9) is defined by

$$
\begin{aligned}
& M(q) \ddot{q}+C(q, \dot{q}) \dot{q}+g(q)=K_{p} \tilde{q}-K_{d} \dot{q}+\xi \\
& \dot{\xi}=K_{i} \tilde{q}
\end{aligned}
$$

Converting the equations above into a matrix form results

in

$$
\frac{d}{d t}\left[\begin{array}{c}
\xi \\
\tilde{q} \\
\dot{\tilde{q}}
\end{array}\right]=\left[\begin{array}{c}
K_{i} \tilde{q} \\
-\dot{q} \\
\ddot{q}^{d}+M^{-1}\left(C \dot{q}+g-K_{p} \tilde{q}+K_{d} \dot{q}-\xi\right)
\end{array}\right]
$$

The equilibrium of $(20)$ is $[\xi, \tilde{q}, \dot{\tilde{q}}]=\left[\xi^{*}, 0,0\right]$. Since at equilibrium point is set as $q=q^{d}$, the equilibrium is defined as $\left[g\left(q^{d}\right), 0,0\right]$. In order to move the equilibrium to the origin, we define

$$
\tilde{\xi}=\xi-g\left(q^{d}\right)
$$

and the closed loop equation becomes

$$
\begin{aligned}
& M(q) \ddot{q}+C(q, \dot{q}) \dot{q}+g(q)=K_{p} \tilde{q}-K_{d} \dot{q}+\tilde{\xi}+g\left(q^{d}\right) \\
& \dot{\tilde{\xi}}=K_{i} \tilde{q}
\end{aligned}
$$

Theorem 1: Since the robot dynamic (9) controlled by linear PID controller (18), the closed loop system (22) is semi-globally asymptotically stable at the equilibrium point $x=\left[\xi-g\left(q^{d}\right), \tilde{q}, \dot{\tilde{q}}\right]^{T}=0$, if the control gains satisfy

$$
\begin{aligned}
& \lambda_{m}\left(K_{p}\right) \geq \frac{3}{2} k_{g} \\
& \lambda_{M}\left(K_{i}\right) \leq \beta \frac{\lambda_{m}\left(K_{p}\right)}{\lambda_{M}(M)} \\
& \lambda_{m}\left(K_{d}\right) \geq \beta+\lambda_{M}(M)
\end{aligned}
$$

where $\beta=\sqrt{\frac{\lambda_{m}(M) \lambda_{m}\left(K_{p}\right)}{3}}, k_{g}$ satisfies (15).

Proof: We construct a Lyapunov function as

$$
\begin{gathered}
V=\frac{1}{2} \dot{q}^{T} M \dot{q}+\frac{1}{2} \tilde{q}^{T} K_{p} \tilde{q}+U(q)-k_{u}+\tilde{q}^{T} g\left(q^{d}\right) \\
+\frac{\alpha}{2} \tilde{\xi}^{T} K_{i}^{-1} \tilde{\xi}+\tilde{q}^{T} \tilde{\xi}+\frac{3}{2} g\left(q^{d}\right)^{T} K_{p}^{-1} g\left(q^{d}\right) \\
-\alpha \tilde{q}^{T} M \dot{q}+\frac{\alpha}{2} \tilde{q}^{T} K_{d} \tilde{q}
\end{gathered}
$$

where $k_{u}=\min _{q}\{U(q)\}, U(q)$ is defined in (8), $k_{u}$ is added such that $V(0)=0 . \alpha$ is a design positive constant. We first prove that $V$ is a Lyapunov function, and $V \geq 0$. The term $\frac{1}{2} \tilde{q}^{T} K_{p} \tilde{q}$ is separated into four parts, such that $V=\sum_{i=1}^{4} V_{i}$

$$
\begin{aligned}
& V_{1}=\frac{1}{6} \tilde{q}^{T} K_{p} \tilde{q}+\tilde{q}^{T} g\left(q^{d}\right)+\frac{3}{2} g\left(q^{d}\right)^{T} K_{p}^{-1} g\left(q^{d}\right) \\
& V_{2}=\frac{1}{6} \tilde{q}^{T} K_{p} \tilde{q}+\tilde{q}^{T} \tilde{\xi}+\frac{\alpha}{2} \tilde{\xi}^{T} K_{i}^{-1} \tilde{\xi} \\
& V_{3}=\frac{1}{6} \tilde{q}^{T} K_{p} \tilde{q}-\alpha \tilde{q}^{T} M \dot{q}+\frac{1}{2} \dot{q}^{T} M \dot{q} \\
& V_{4}=U(q)-k_{u}+\frac{\alpha}{2} \tilde{q}^{T} K_{d} \tilde{q}^{2} \geq 0
\end{aligned}
$$

It is possible to show that

$$
V_{1}=\frac{1}{2}\left[\begin{array}{c}
\tilde{q} \\
g\left(q^{d}\right)
\end{array}\right]^{T}\left[\begin{array}{cc}
\frac{1}{3} K_{p} & I \\
I & 3 K_{p}^{-1}
\end{array}\right]\left[\begin{array}{c}
\tilde{q} \\
g\left(q^{d}\right)
\end{array}\right] \geq 0
$$

When $\alpha \geq \frac{3}{\lambda_{m}\left(K_{i}^{-1}\right) \lambda_{m}\left(K_{p}\right)}$,

$$
\begin{aligned}
& V_{2} \geq \frac{1}{2} \frac{1}{6} \lambda_{m}\left(K_{p}\right)\|\tilde{q}\|^{2}-\|\tilde{q}\|\|\tilde{\xi}\|+\frac{\alpha \lambda_{m}\left(K_{i}^{-1}\right)}{2}\|\tilde{\xi}\|^{2} \\
& =\frac{1}{2}\left(\sqrt{\frac{1}{3} \lambda_{m}\left(K_{p}\right)}\|\tilde{q}\|-\sqrt{\frac{3}{\lambda_{m}\left(K_{p}\right)}}\|\tilde{\xi}\|\right)^{2} \geq 0
\end{aligned}
$$

Because

$$
y^{T} A x \leq\|y\|\|A x\| \leq\|y\|\|A\|\|x\| \leq\left|\lambda_{M}(A)\right|\|y\|\|x\|
$$

when $\alpha \leq \frac{\sqrt{\frac{1}{3} \lambda_{m}(M) \lambda_{m}\left(K_{p}\right)}}{\lambda_{M}(M)}$,

$$
\begin{aligned}
& V_{3} \geq \frac{1}{2}\left(\lambda_{m}(M)\|\dot{q}\|^{2}-2 \alpha \lambda_{M}(M)\|\tilde{q}\|\|\dot{q}\|\right. \\
& \left.+\frac{1}{3} \lambda_{m}\left(K_{p}\right)\|\tilde{q}\|^{2}\right) \\
& =\frac{1}{2}\left(\sqrt{\lambda_{m}(M)}\|\dot{q}\|-\sqrt{\frac{1}{3} \lambda_{m}\left(K_{p}\right)}\|\tilde{q}\|\right)^{2} \geq 0
\end{aligned}
$$

Obviously, if

$$
\sqrt{\frac{1}{3}} \lambda_{m}\left(K_{i}^{-1}\right) \lambda_{m}^{\frac{3}{2}}\left(K_{p}\right) \lambda_{m}^{\frac{1}{2}}(M) \geq \lambda_{M}(M)
$$

there exists

$$
\frac{\sqrt{\frac{1}{3} \lambda_{m}(M) \lambda_{m}\left(K_{p}\right)}}{\lambda_{M}(M)} \geq \alpha \geq \frac{3}{\lambda_{m}\left(K_{i}^{-1}\right) \lambda_{m}\left(K_{p}\right)}
$$

This means that if $K_{p}$ is sufficiently large or $K_{i}$ is sufficiently small, (30) is established, and $V(\dot{q}, \tilde{q}, \tilde{\xi})$ is globally positive definite.

Taking the derivative of $V$, we get

$$
\begin{aligned}
& \dot{V}=\dot{q}^{T} M \ddot{q}+\frac{1}{2} \dot{q}^{T} \dot{M} \dot{q}+\dot{\tilde{q}}^{T} K_{p} \tilde{q}+g(q)^{T} \dot{q}+\dot{\tilde{q}}^{T} g\left(q^{d}\right) \\
& +\alpha \tilde{\xi}^{T} K_{i}^{-1} \tilde{\xi}+\dot{\tilde{q}} \tilde{\xi}+\tilde{q}^{T} \dot{\tilde{\xi}} \\
& -\alpha\left(\dot{\tilde{q}} M \dot{q}+\tilde{q}^{T} \dot{M} \dot{q}+\tilde{q}^{T} M \ddot{q}\right)-\alpha \tilde{q}^{T} K_{d} \dot{q}
\end{aligned}
$$

Using (13), $\frac{d}{d t} U\left(\underset{T}{q)}=\dot{q}^{T} g(q), \frac{d}{d t} g\left(q^{d}\right)=0\right.$ and $\frac{d}{d t}\left[\tilde{q}^{T} g\left(q^{d}\right)\right]=\dot{\tilde{q}} g\left(q^{d}\right)$, the first three terms of (32) become

$$
-\dot{q}^{T} g(q)-\dot{q}^{T} K_{d} \dot{q}+\dot{q}^{T} \tilde{\xi}+\dot{q}^{T} g\left(q^{d}\right)
$$

Because $\dot{\tilde{q}}^{T} g\left(q^{d}\right)=-\dot{q}^{T} g\left(q^{d}\right)$ and $\dot{\tilde{\xi}}=K_{i} \tilde{q}$, the first seven terms of (32) are

$$
-\dot{q}^{T} K_{d} \dot{q}+\alpha \tilde{q}^{T} \tilde{\xi}+\tilde{q}^{T} K_{i} \tilde{q}
$$

Now we focus our attention on the last term of (32). From (14), we have

$$
\tilde{q}^{T} \dot{M} \dot{q}=\tilde{q}^{T} C \dot{q}+\tilde{q}^{T} C^{T} \dot{q}
$$

From (9)

$$
\begin{gathered}
\tilde{q}^{T} M \ddot{q}=-\tilde{q}^{T} C \dot{q}-\tilde{q}^{T} g(q)+\tilde{q}^{T} K_{p} \tilde{q}-\tilde{q}^{T} K_{d} \dot{q} \\
+\tilde{q}^{T} \tilde{\xi}+\tilde{q}^{T} g\left(q^{d}\right)
\end{gathered}
$$


.$T$

Since $\dot{\tilde{q}} M \dot{q}=-\dot{q}^{T} M \dot{q}$, using (12) and (15) the last two terms of (32) are

$$
\begin{aligned}
& -\alpha\left\{\tilde{q}^{T} K_{p} \tilde{q}-\dot{q}^{T} M \dot{q}+\tilde{q}^{T} C^{T} \dot{q}\right. \\
& \left.+\tilde{q}^{T}\left[g\left(q^{d}\right)-g(q)\right]+\tilde{q}^{T} \tilde{\xi}\right\} \\
& \leq \alpha \dot{q}^{T} M \dot{q}-\alpha \tilde{q}^{T} K_{p} \tilde{q}+\alpha k_{c}\|\tilde{q}\|\|\dot{q}\|^{2} \\
& +\alpha k_{g}\|\tilde{q}\|^{2}-\alpha \tilde{q}^{T} \tilde{\xi}
\end{aligned}
$$

From (34) and (37)

$$
\begin{aligned}
& \dot{V} \leq-\dot{q}^{T}\left(K_{d}-\alpha M-\alpha k_{c}\|\tilde{q}\|\right) \dot{q} \\
& -\tilde{q}^{T}\left(\alpha K_{p}-K_{i}-\alpha k_{g}\right) \tilde{q} \\
& \leq-\left[\lambda_{m}\left(K_{d}\right)-\alpha \lambda_{M}(M)-\alpha k_{c}\|\tilde{q}\|\right]\|\dot{q}\|^{2} \\
& -\left[\alpha \lambda_{m}\left(K_{p}\right)-\lambda_{M}\left(K_{i}\right)-\alpha k_{g}\right]\|\tilde{q}\|^{2}
\end{aligned}
$$

If

$$
\|\tilde{q}\| \leq \frac{\lambda_{M}(M)}{\alpha k_{c}}
$$

and

$$
\begin{aligned}
& \lambda_{m}\left(K_{d}\right) \geq(1+\alpha) \lambda_{M}(M) \\
& \lambda_{m}\left(K_{p}\right) \geq \frac{1}{\alpha} \lambda_{M}\left(K_{i}\right)+k_{g}
\end{aligned}
$$

then $\dot{V} \leq 0,\|\tilde{q}\|$ decreases. $>$ From (31), if

$$
\begin{aligned}
& \lambda_{m}\left(K_{d}\right) \geq \lambda_{M}(M)+\sqrt{\frac{1}{3} \lambda_{m}(M)} \sqrt{\lambda_{m}\left(K_{p}\right)} \\
& \lambda_{m}\left(K_{p}\right) \geq \frac{1}{3} \lambda_{m}\left(K_{i}^{-1}\right) \lambda_{m}\left(K_{p}\right) \lambda_{M}\left(K_{i}\right)+k_{g}
\end{aligned}
$$

then (40) is established. Using (30) and $\lambda_{m}\left(K_{i}^{-1}\right)=$ $\frac{1}{\lambda_{M}\left(K_{i}\right)},(41)$ is $(23)$.

$\dot{V}$ is negative semi-definite. Define a ball $\Sigma$ of radius $\sigma>$ 0 centered at the origin of the state space, which satisfies these condition

$$
\Sigma=\left\{\tilde{q}:\|\tilde{q}\| \leq \frac{\lambda_{M}(M)}{\alpha k_{c}}=\sigma\right\}
$$

$\dot{V}$ is negative semi-definite on the ball $\Sigma$. There exists a ball $\Sigma$ of radius $\sigma>0$ centered at the origin of the state space for which $\dot{V} \leq 0$. The origin of the closed-loop equation (22) is a stable equilibrium. Since the closed-loop equation is autonomous, we may use La Salle's theorem. Define $\Omega$ as

$$
\begin{aligned}
& \Omega=\left\{x(t)=[\tilde{q}, \dot{q}, \tilde{\xi}] \in R^{3 n}: \dot{V}=0\right\} \\
& =\left\{\tilde{\xi} \in R^{n}: \tilde{q}=0 \in R^{n}, \dot{q}=0 \in R^{n}\right\}
\end{aligned}
$$

From (32), $\dot{V}=0$ if and only if $\tilde{q}=\dot{q}=0$. For a solution $x(t)$ to belong to $\Omega$ for all $t \geq 0$, it is necessary and sufficient that $\tilde{q}=\dot{q}=0$ for all $t \geq 0$. Therefore it must also hold that $\ddot{q}=0$ for all $t \geq 0$. We conclude that from the closed-loop system (22), if $x(t) \in \Omega$ for all $t \geq 0$, then

$$
\begin{aligned}
& g(q)=g\left(q^{d}\right)=\tilde{\xi}+g\left(q^{d}\right) \\
& \dot{\tilde{\xi}}=0
\end{aligned}
$$

implies that $\tilde{\xi}=0$ for all $t \geq 0$. So $x(t)=[\tilde{q}, \dot{q}, \tilde{\xi}]=0 \in$ $R^{3 n}$ is the only initial condition in $\Omega$ for which $x(t) \in \Omega$ for all $t \geq 0$.

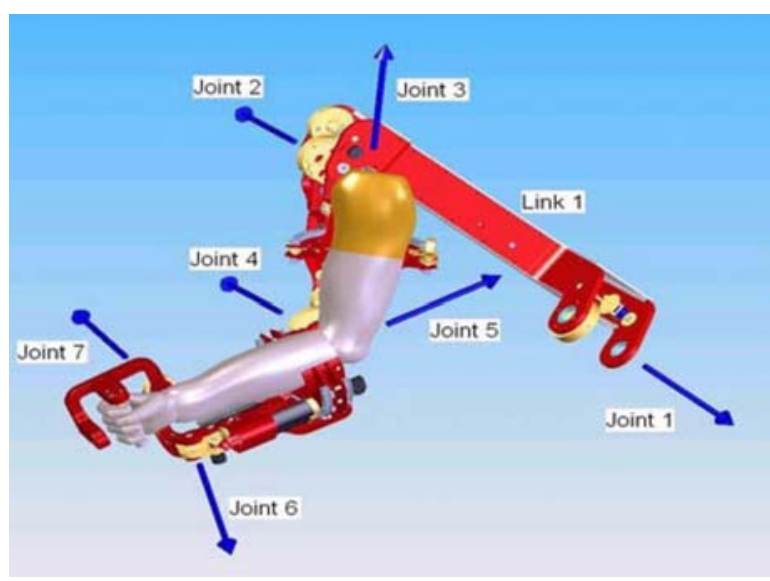

Fig. 3. Exoskeletal axes assignment in relation to the human arm.

Finally, we may conclude that the origin of the closedloop system (22) is locally asymptotically stable. Because $\frac{1}{\alpha} \leq \lambda_{m}\left(K_{i}^{-1}\right) \lambda_{m}\left(K_{p}\right)$, the upper bound for $\|\tilde{q}\|$ can be

$$
\tilde{q} \leq \frac{\lambda_{M}(M)}{k_{c}} \lambda_{M}\left(K_{i}\right) \lambda_{m}\left(K_{p}\right)
$$

It establishes the semi-global stability of our controller, in the sense that the domain of attraction can be arbitrarily enlarged with a suitable choice of the gains. Namely, for increasing $K_{p}$ the basin of attraction will grow as well.

Remark 1: Based on the above stability analysis, we may conclude that the three gain matrices of the linear PID control (18) can be chosen directly from the conditions (23). The most important contribution of the proposed method is that the PID parameters can be calculated directly without an exact model of the plant, resulting in a simpler PID controller design as opposed to the sate of the art experimental tuning procedures in [1][2][19][21][20][24][30]. This linear PID control is exact the same as the industrial robot controllers, and is semi-globally asymptotically stable.

\section{Simulation Results}

The joint axes of the 7-DOF upper limb Exoskeleton (EXO-UL7) are depicted in Figure 3 in which Joint 1 is shoulder abduction-adduction, Joint 2 is shoulder exion extension, Joint 3 is shoulder internal-external rotation, Joint 4 is elbow exion extension, Joint 5 is forearm pronationsupination, Joint 6 is wrist extension, and Joint 7 is wrist radial-ulnar deviation.

Given a linear PID control configuration (18), along and conditions (23) are used to determine the controller parameters. The joint velocities are estimated by the standard filters [37]

$$
\widetilde{\dot{q}}(s)=\frac{3 s}{0.1 s+3} q(s)
$$

The structural properties of the EXO-UL7 with respect to base frame are shown in Table 2. 

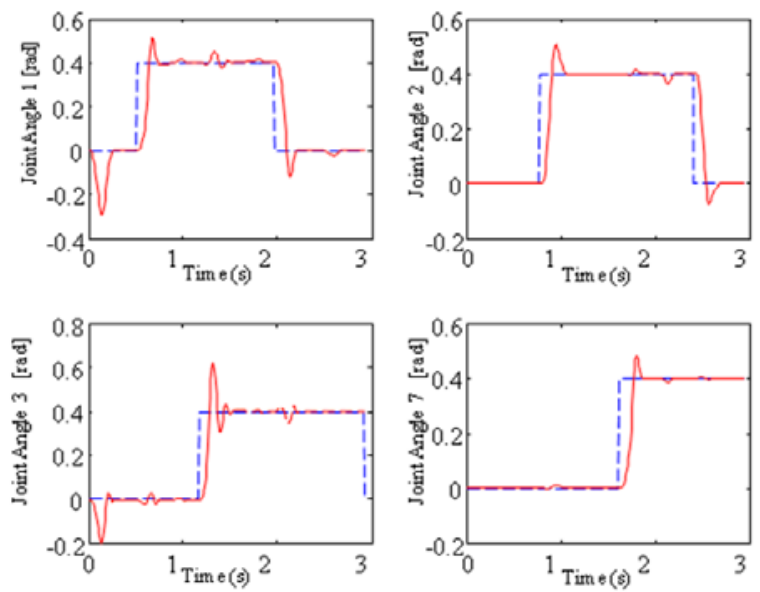

Fig. 4. Simulation results of the EXO-UL7 upper limb exoskeleton response to a step input using the PID control for joints 1, 2,4,and 7 .

Table 2. Parameters of the EXO-UL7

\begin{tabular}{|l||l|l|l|l|}
\hline Joint & Mass $(\mathrm{Kg})$ & Center of Mass $(\mathrm{m})$ & Link Length $(\mathrm{m})$ & Joint Offset $(\mathrm{m})$ \\
\hline \hline 1 & 3.4 & 0.3 & 0.7 & 0.3 \\
\hline 2 & 1.7 & 0.05 & 0.1 & 0 \\
\hline 3 & 0.7 & 0.1 & 0.2 & 0.1 \\
\hline 4 & 1.2 & .02 & 0.05 & 0 \\
\hline 5 & 1.8 & .02 & 0.05 & 0.1 \\
\hline 6 & 0.2 & 0.04 & 0.1 & 0 \\
\hline 7 & 0.5 & 0.02 & 0.05 & 0.1 \\
\hline
\end{tabular}

The above parameters are used to estimate the upper and lower bounds of the inertia matrix $M(q)$ eigenvalues along with $k_{g}$ in (15). The computed values are $\lambda_{M}(M)=5$, $\lambda_{m}(M)=0.1, k_{g}=10$,

$$
\begin{aligned}
& K_{p}=\operatorname{diag}[200,100,80,150,50,50,50] \\
& K_{i}=\operatorname{diag}[12,3,5,11,2,3,3] \\
& K_{d}=\operatorname{diag}[30,13,15,20,12,13,13]
\end{aligned}
$$

where $\beta=1.3$, and $\lambda_{m}\left(K_{p}\right)=50>15, \lambda_{M}\left(K_{i}\right)=12<$ $13, \lambda_{m}\left(K_{d}\right)=12>6.3$.

Simulation results of Joint 1, Joint 2, Joint 4 and Joint 7 with the parameters in (47) are shown in Figure 4 and Figure 5. The condition (23) in Theorem 1 provides necessary conditions for selecting PID parameters. If $K_{p, 2}$ is changed from it initial value of 80 to 20 , or $K_{i, 4}$ is changed from it initial value 11 to 20 , or $K_{d, 1}$ is changed from from it initial value of 30 to 5 , the closed-loop system becomes unstable. However, the the orthogonal parameters of the PID controller in (47) satisfying the condition (23), leads to closed-loop system that is asymptotically stable, but these parameters are by no means the optimal set.

\section{CONCLUSiOnS}

A novel linear PID control for a class of wearable robotic manipulators is addressed. The conditions of the semi-global asymptotic stability were defined for a common linear PID control architecture. The advantage of the proposed approach is that a full mathematical model of the system is not
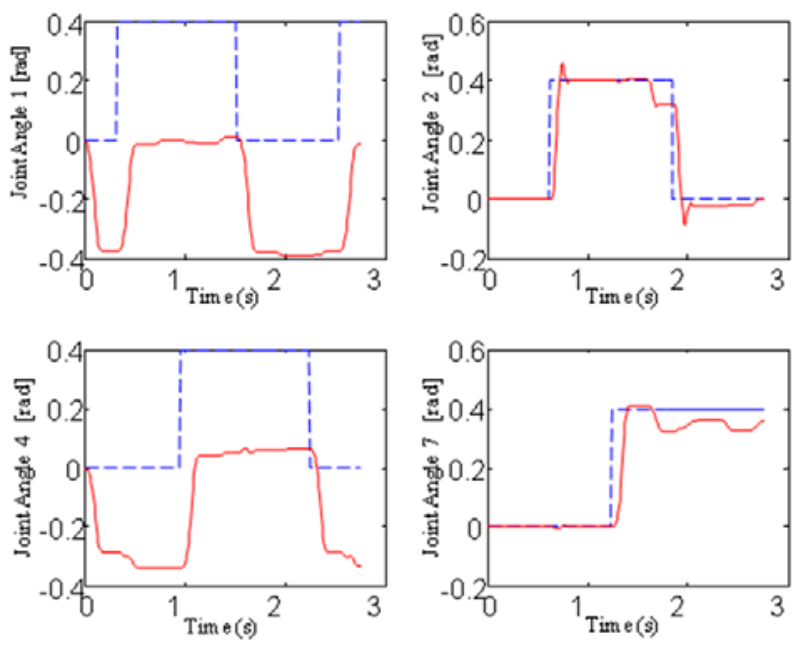

Fig. 5. Simulation results of the EXO-UL7 upper limb exoskeleton response to a step input using a PD control for joints 1, 2,4,and 7.

required for selecting the parameters of the PID controller. The simulation results using EXO-UL7 system validated the proposed design methodology of the PID control. It is important to note that the proposed methodology does not lead to the optimal set of a linear PID controller parameters. However this initial set of parameters satisfying the semiglobal asymptotic stability may be further used as the initial set of further optimization once a unique design criteria are defined.

\section{REFERENCES}

[1] J.Alvarez-Ramirez, R.Kelly, I.Cervantes, Semiglobal stability of saturated linear PID control for robot manipulators, Automatica, vol.39,989-995, 2003

[2] S. Arimoto, Fundamental problems of robot control: Part I, Innovations in the realm of robot servo-loops, Robotica, vol.13, No.1, 19-27, 1995.

[3] Banala SK, Kulpe A, Agrawal SK. A powered leg orthosis for gait rehabilitation of motor-impaired patients. IEEE International Conference of Robotics and Automation, April 2007. PDF; Rome, Italy.2007.

[4] Blaya A, Herr H. Adaptive control of a variable Impedance ankle-foot orthosis to assist drop-foot gait. IEEE Trans Neural Syst Rehabil Eng. 12(1):24-31, 2004

[5] Bergamasco M, Frisoli A, Avizzano CA. Exoskeletons as manmachine interface systems for teleoperation and interaction. Advanced in telerobotics. Berlin/Heidelberg: Springer, 2007

[6] Carignan CR, Naylor MP, Roderick SN. Controlling shoulder impedance in a rehabilitation arm exoskeleton, IEEE International Conference on Robotics and Automation, Pasadena; May 19-23, California, USA, 2008

[7] Casolo F, Cinquemani S, Cocetta M. 2008. On active lower limb exoskeletons actuators. Proceedings of the 5th International Symposium onMechatronics and its Applications (ISMA08); 2008 May 27-29; Amman, Jordan.

[8] A.M. Dollar, and H.Herr, Lower Extremity Exoskeletons and Active Orthoses: Challenges and State-of-the-Art, IEEE Transactions on Robotics, Volume 24, No. 1, pp. 1-15, August 2008

[9] Ferris DP, Sawicki GS, Daley MA. A physiologist's perspective on robotic exoskeletons for human locomotion. Int $J$ Humanoid Rob. 4:507-528. 2007

[10] L.Greenemeier, Trouble walking? Try Honda's new exoskeleton legs, Scientific American., November 10, 2008

[11] E.Guizzo , H.Goldstein, The rise of the body bots, IEEE Spectrum, 42(10):50-56. 2005. 
[12] H.Herr, Exoskeletons and orthoses: classification, design challenges and future directions, Journal of NeuroEngineering and Rehabilitation, 6(21) 2009.

[13] N.Hogan, Skeletal muscle impedance in the control of motor actions, Journal of Mechanics in Medicine an Biology, 2(3), 359-373, 2002.

[14] Kawamoto H, Kanbe S, Sankai Y. Power assist method for HAL-3 estimating operator's intention based on motion information. Proceedings of the 2003 IEEE InternationalWorkshop on Robot and Human Interactive Communication; Millbrae, CA, USA. 2003.

[15] H.Kazerooni and H. Ming-Gau, The dynamics and control of a haptic interface device, IEEE Transactions on Robotics and Automation, 10(4), 453-464, 1994.

[16] H.Kazerooni, R.Steger, The Berkeley lower extremity exoskeleton, Journal of Dynamic Systems, Measurements, and Control-Transactions of the ASME, Vol.128, 14-25, 2006

[17] Kiguchi K, Rahman M, Sasaki M. 2006. Neuro-fuzzy-based motion control of a robotic exoskeleton: considering endeffector force vectors. ICRA 2006; Orlando, FL, USA. 3146-3151

[18] R.Kelly, PD control with desired gravity compensation of robotic manipulators: A review, International Journal of Robotics Research, Vol. 16, No.5, pp. 660-672, 1997

[19] R.Kelly, Global Positioning of Robot Manipulators via PD Control Plus a Class of Nonlinear Integral Actions, IEEE Transactions on Automatic Control, Vol.43, No.7, 934-938, 1998

[20] R.Kelly, V.Santibáñez, L.Perez, Control of Robot Manipulators in Joint Space, Springer-Verlag London, 2005.

[21] R.Kelly, A tuning procedure for stable PID control of robot manipulators, Robotica, $13: 141-148,1995$

[22] Klein J, et al., Biomimetic orthosis for the neurorehabilitation of the elbow and shoulder (BONES). IEEE Biorob, 2008, Scottsdale, AZ, USA. 535-541

[23] Mihelj M, Nef T, Riener R., ARMin II-7 DoF rehabilitation robot: mechanics and kinematics. ICRA 2007; Rome, Italy. 4120-4125.

[24] E.V. L. Nunes, L.Hsu, F.Lizarralde, Arbitrarily small damping allows global output feedback tracking of a class of Euler-Lagrange systems, 2008 American Control Conference, , Seattle, USA, 378-382, 2008

[25] V.Parra-Vega, S.Arimoto, Y.-H.Liu,G.Hirzinger, P.Akella, Dynamic Sliding PID Control for Tracking of Robot Manipulators: Theory and Experiments, IEEE Transactions on Robotics and Automation, Vol.19, No.6, 967-976, 2003

[26] B.Paden, R.Panja, Globally asymptotically stable PD + controller for robot manipulators, International Journal of Control, Vol. 47, No. 6 , $1697-1712,1988$

[27] Perry Joel, Janet Powell, Jacob Rosen, Isotropy of an Upper Limb Exoskeleton and the Kinematics and Dynamics of the Human Arm, Journal of Applied Bionics and Biomechanics, Vol. 6, No. 2, pp. 175191, June 2009

[28] Perry Joel C., Jacob Rosen, Design of a 7 Degree-of-Freedom UpperLimb Powered Exoskeleton, Proceedings of the 2006 BioRob Conference, Pisa, Italy, February, 2006.

[29] Pons JL. Wearable robots: biomechatronic exoskeletons. San Francisco, CA: Wiley. 2007

[30] P.Rocco, Stability of PID control for industrial robot arms, IEEE Transactions on Robotics and Automation, VOL.12, NO. 4 , 606$614,1996$.

[31] Rosen Jacob, and J.C. Perry, Upper Limb Powered Exoskeleton, Journal of Humanoid Robotics, Vol. 4, No. 3 (2007) 1-20

[32] Rosen Jacob, Joel C. Perry, Nathan Manning, Stephen Burns, Blake Hannaford, The Human Arm Kinematics and Dynamics During Daily Activities: Toward a 7 DOF Upper Limb Powered Exoskeleton, 2005 International Conference on Advanced Robotics (ICAR 2005), Seattle WA, July 2005.

[33] Romilly DP, Anglin C, Gosine RG, Hershler C, Raschke SU. A functional task analysis and motion simulation for the development of a powered upper-limb orthosis. IEEE Trans Rehabil Eng. 2(3):119129,1994

[34] Z.Qu, D.M. Dawson, S. Y. Lim, J.F. Dorsey, A New Class of Robust Control Laws for Tracking of Robots, International Journal of Robotics Research, Vol. 13, No.4, 355-363, 1994

[35] Stienen AHA, Hekman EEG, Van der Helm FCT, Prange GB, Jannink MJA, Aalsma AMM, Van der Kooij H. Dampace: dynamic forcecoordination trainer for the upper extremities. IEEE 10th International Conference on Rehabilitation Robotics (ICORR) 2007; Kyoto, Japan

[36] J. J.Slotine, W.Li, Adaptive manipulator control: A case study, IEEE Transactions on Automatic Control, Vol.33, No.11, 995-1003, 1988
[37] M.W.Spong and M.Vidyasagar, Robot Dynamics and Control, John Wiley \& Sons Inc., Canada, 1989.

[38] Tsagarakis NG, Caldwell DG. 2003. Development and control of a "Soft-Actuated" exoskeleton for use in physiotherapy and training. Auton Robots. 15(1):21-33.

[39] W.Truccolo, G.M.Friehs, J.P.Donoghue, L.R.Hochberg, Primary motor cortex tuning to intended movement kinematics in humans with tetraplegia, J Neurosci, 28(5), 1163-1178, 2008

[40] P.Tomei, Adaptive PD Controller for Robot Manipulators, IEEE Transactions on Robotics and Automation, VOL. 7, NO. 4 , 565-570,1991.

[41] Van der Loos HFM, Reinkensmeyer DJ. Rehabilitation and health care robotics, Handbook of Robotics. New York, NY: Springer; p. 1223 1251, 2008.

[42] Zoss A, Kazerooni H, Chu A. On the mechanical design of the Berkeley lower extremity exoskeleton (BLEEX). Proceedings of IEEE International Conference on Robotics and Automation, Barcelona, Spain. April 18-22, 2005. 